\title{
Avaliação da Resiliência: Controvérsia em Torno do Uso das Escalas
}

\author{
Resilience Assessment: Controversies about the Use of Scales
}

\author{
Caroline Tozzi Reppold ${ }^{*}$, , Jeferson Charles Mayer ${ }^{a}$, Leandro Silva Almeida ${ }^{b}$ \\ \& Claudio Simon Hutz ${ }^{c}$ \\ ${ }^{a}$ Universidade Federal de Ciências da Saúde de Porto Alegre, Porto Alegre, Brasil, \\ ${ }^{b}$ Universidade do Minho, Braga, Portugal \& ${ }^{c}$ Universidade Federal do Rio Grande do Sul, Porto Alegre, Brasil
}

\begin{abstract}
Resumo
Resiliência é um construto que descreve a capacidade de um indivíduo superar, com relativo sucesso, condições adversas ou situações que envolvem risco ao seu bem-estar, desenvolvimento e saúde mental. Alguns pesquisadores da área interpretam essa definição como uma indicação de que a resiliência é similar a outras variáveis de ajustamento e presumem a sua estabilidade temporal. Isso leva sua avaliação a ser feita frequentemente através de escalas autoadministradas, por vezes questionáveis em termos de sua precisão e validade. Este artigo discute a abrangência e o histórico da resiliência psicológica e as várias formas de sua avaliação. Mais especificamente, o objetivo deste artigo é apresentar argumentos contra a pertinência da utilização de escalas de autorrelato para a mensuração da resiliência, considerando-se o caráter relacional, processual e contextual desse construto. Para tanto, instrumentos disponíveis na literatura para avaliação da resiliência são revisados e discutidos, apontando orientações para a pesquisa na área. Palavras-chave: Resiliência, avaliação psicológica, mensuração.
\end{abstract}

\begin{abstract}
Resilience is a construct which describes the capacity of individuals to overcome, with relative success, adverse conditions or risky situations to their well-being, development, and mental health. Some researchers interpret this definition as an indication that resilience is similar to some other adjustment variables and they assume that it presents temporal stability. Resilience is frequently assessed using self-report scales, which may also present validity and reliability problems. The present paper discusses the history and the comprehensiveness of psychological resilience and the different ways of evaluating it. More specifically, the objective of this study is to present arguments against the use of self-report scales to measure resilience, because of the relational, processual and contextual character of such construct. The main instruments available in the literature are reviewed and discussed and new directions for research in the area are suggested.

Keywords: Resilience, psychological assessment, measuring.
\end{abstract}

No campo das ciências comportamentais, o conceito de resiliência refere-se essencialmente a um processo de adaptação positiva diante de um contexto tipicamente desfavorável, no qual o indivíduo demonstra uma notável capacidade de superação de condições adversas que representariam uma ameaça significativa ao seu bemestar, desenvolvimento ou saúde mental (Luthar, 2006; Masten, 2007; Rutter, 2006). Ao longo de décadas, o estudo científico da resiliência vem apresentando uma evolução considerável, marcada não apenas pela elaboração de fundamentos teóricos e princípios metodológicos, ou

* Endereço para correspondência: Universidade Federal de Ciências da Saúde de Porto Alegre, Sarmento Leite, 245, sala 610, Centro, Porto Alegre, RS, Brasil 90050170. E-mail: carolinereppold@yahoo.com.br e claudio.hutz@terra.com.br pela reunião de um amplo conjunto de evidências que suportam sua consolidação como um construto psicológico de grande capacidade heurística na Psicologia, mas também pelo surgimento de vários questionamentos em torno das dimensões e das medidas relativas a esse construto (Masten \& Obradovié, 2006). Tais questionamentos acabam por refletir a relevância prática e o elevado interesse da comunidade científica em relação a esse tema, representando uma contribuição fundamental para o seu progressivo refinamento epistemológico.

Uma das áreas em que se manifesta a controvérsia existente prende-se à mensurabilidade psicológica da resiliência. As formas tradicionalmente utilizadas para avaliar esse construto podem ser classificadas nas seguintes categorias: (a) escores de testes e outras medidas de desempenho, como, por exemplo, notas escolares; (b) perfis obtidos por questionários de personalidade ou 
temperamento; (c) entrevistas e análise de histórias de vida; e (d) entrevistas e análise do discurso de pessoas informantes que convivem com o sujeito. Contudo, visando a obter uma forma mais universal e objetiva de avaliar esse construto, alguns pesquisadores desenvolveram instrumentos de avaliação psicológica voltados especificamente para a expressão de uma medida quantitativa do grau de resiliência. Incluem-se nessa categoria as conhecidas escalas de resiliência, por exemplo: Resilience Scale (Wagnild \& Young, 1993), Connor-Davidson Resilience Scale (CD-RISC; Connor \& Davidson, 2003) e Adolescent Resilience Scale (Oshio, Kaneko, Nagamine, \& Nakaya, 2003).

Essas escalas, optando por indicadores facturais na formulação dos itens e solicitando aos sujeitos uma resposta diferida assente em sua percepção cognitiva, têm sido objeto de alguma contestação. Para alguns autores, a adequação de instrumentos desse tipo deve ser questionada, dada a natureza dinâmica, contextual e multidimensional da resiliência, a qual não corresponde simplesmente a um traço pessoal, mas sim a um processo transacional intrinsecamente complexo de aprendizagem e de desenvolvimento, que não pode ser apropriadamente expresso através de uma medida escalar e momentânea (Masten \& Obradovié, 2006; Rutter, 2007).

Assim, o objetivo do presente artigo é discutir a pertinência da criação e utilização de escalas para mensuração da resiliência, considerando-se o caráter relacional, desenvolvimental e contextual desse construto. Para tanto, são discutidos os processos envolvidos nas situações em que se diz que o indivíduo é resiliente e procura-se demonstrar que, para a compreensão da resiliência, é necessário ir além de uma simples avaliação dos fatores de risco, proteção e autoconfiança envolvidos na situação.

\section{Perspectiva Histórica}

Desde a segunda metade do século XX, diversos pesquisadores têm realizado estudos relacionados ao desenvolvimento humano e à associação entre a ocorrência de transtornos mentais e a exposição do indivíduo a fatores de risco, eventos estressores ou outras adversidades biopsicossociais. A partir dos resultados obtidos por esses estudos, observa-se a existência de uma grande heterogeneidade entre os padrões de resposta de diferentes indivíduos e culturas a tais adversidades, variando desde a ocorrência de psicopatologias graves e persistentes, até a ausência de quaisquer evidências de prejuízos significativos (Rutter, 2000, 2007).

Um dos trabalhos pioneiros mais importantes nessa linha de pesquisa foi o estudo prospectivo de coorte conhecido como Kauai Longitudinal Study (Werner, Bierman, \& French, 1971; Werner \& Smith, 1977, 1982, 1992), iniciado em 1955 por uma equipe interdisciplinar de profissionais da saúde, com o objetivo de acompanhar o desenvolvimento de 698 crianças nascidas naquele ano na ilha de Kauai (Havaí). Os participantes foram periodicamente avaliados, desde o período perinatal até a idade adulta (ao completar 1, 2, 10, 18 e 32 anos), buscando-se analisar as consequências, em longo prazo, da exposição a fatores de risco diversos. Inicialmente, o estudo concentrou-se na avaliação da vulnerabilidade dos indivíduos em relação a condições desfavoráveis comumente presentes na população estudada, tais como estresse perinatal, dificuldades socioeconômicas, psicopatologias parentais e rupturas da unidade familiar.

Os instrumentos utilizados nesses estudos foram: $\mathrm{CoO}-$ perative School and College Ability Tests, aplicados para avaliação de habilidades verbais; Sequential Tests of Educational Progress, para avaliação de leitura, escrita e matemática; The California Psychological Inventory e Nowicki Strickland Locus of Control Scale, destinados à avaliação de características de personalidade. Questionários biográficos que continham informações sobre status educacional, vocacional e matrimonial dos pais das crianças foram aplicados para avaliação infantil, assim como questionários investigando saúde e eventos estressores ao longo da adolescência e da idade adulta. A avaliação foi ainda complementada pelos resultados obtidos nos testes Cattel Infant Intelligence Scale (destinado à avaliação de medidas sensório-motoras de ajustamento emocional e de habilidades de linguagem); Primary Mental Abilities Test (PMA), Bender-Gestalt Test e Vineland Social Maturity Scale (mais voltados para a avaliação de diversas facetas das habilidades e competências cognitivas). Nessas medidas, indivíduos resilientes obtiveram, em geral, escores significativamente mais altos do que as crianças do grupo controle. A avaliação do ambiente familiar incluiu, em todas as etapas, medidas sobre as seguintes variáveis: nível educacional materno (baseado no número de anos de escolaridade), status socioeconômico (baseado na ocupação paterna, padrão de vida, condições de habitação e quantidade de moradores na residência) e estabilidade familiar (baseada nas informações das entrevistas, presença ou ausência do pai, alcoolismo, problemas de saúde mental dos pais e discordâncias entre os pais).

Para a maioria das variáveis investigadas, à medida que as pesquisas progrediram, verificou-se que alguns indivíduos, apesar de estarem expostos a sérios fatores de risco, conseguiram superar dificuldades significativas, tornando-se jovens adultos positivamente adaptados, competentes e autônomos, enquanto outros apresentavam um padrão diferente de desenvolvimento e de adaptação. A partir desta constatação, o foco do estudo voltou-se progressivamente para a identificação e análise dos elementos que provavelmente estariam relacionados ao grau de capacidade de superação desses indivíduos (Werner, 1993; Werner \& Smith, 1982)

Nessa linha, estudos prospectivos conduzidos por E. James Anthony, publicados na década de 70, investigaram o impacto sofrido por crianças cujos pais apresenta- 
vam transtornos psicopatológicos. Os resultados demonstraram que, apesar do risco genético e ambiental aos quais estavam submetidas, algumas dessas crianças não haviam sofrido prejuízos significativos, apresentando boa saúde psicológica e elevado nível de competência (Anthony, 1974, 1987). Em referência a essa demonstração de resistência diante de um contexto de risco, Anthony passou a utilizar o termo "invulnerabilidade", o qual seria posteriormente refutado por transmitir uma ideia inadequada de que tal adaptação positiva decorria de uma condição absoluta e imutável, ou ainda a uma característica inerente apenas a fatores constitucionais do indivíduo (Rutter, 2000).

No estudo de Anthony (1987) utilizou-se, para avaliação psicológica da vulnerabilidade, uma escala quantitativa que cobria diversas áreas do funcionamento psicológico. As 14 variáveis testadas incluíram: presença e severidade de transtorno psicológico, lógica de pensamento, realidade, concretude, eficiência na defesa, estratégias de coping, conteúdo de patologia, ansiedade, identificação, controle do impulso, adaptação emocional e agressividade. Cada díade mãe/filho respondeu também ao General Information Questionnaire (GIQ; Janes, Gilpin, Lander, \& Finn, 1975), um questionário referente a informações sobre o padrão de vida dos filhos e de seus pais, no que compete ao funcionamento escolar e laboral, relações familiares, participação social e relacionamento com a comunidade; e ao Rochester Adaptive Behavior Inventory (Jones, 1977), uma entrevista com as mães, envolvendo aspectos acadêmicos e sociais dos filhos, bem como a qualidade das relações familiares. O Minnesota Multiphasic Personality Inventory (MMPI; Hathaway \& McKinney, 1942) foi utilizado, ainda, para avaliação de personalidade. Portanto, seja no estudo do grupo de Werner, seja no estudo de Anthony, os instrumentos utilizados na pesquisa avaliavam variáveis envolvidas na resiliência, mas não havia um instrumento específico destinado à mensuração direta desse construto.

Também na década de 1970, Norman Garmezy, professor da Universidade de Minnesota, fundou um programa de pesquisa denominado Project Competence, com o objetivo de estudar processos adaptativos e indicadores de competência em crianças sujeitas a diferentes adversidades, a partir de uma perspectiva desenvolvimental. Um dos diversos trabalhos originados a partir desse projeto foi um estudo prospectivo de coorte de uma amostra normativa de 205 crianças escolares da cidade de Minneapolis (Minnesota), que foram posteriormente reavaliadas quando decorridos 7, 10 e 20 anos desde a realização de sua avaliação inicial. Ao longo do tempo, esse estudo foi sendo acompanhado por outros estudos concomitantes do Project Competence, especificamente focados em amostras de risco, as quais incluíram crianças que apresentavam deficiências físicas, problemas cardíacos congênitos e históricos de psicopatologia parental, além de crianças moradoras de abrigos e refugiadas de guerra (Masten,
Morison, Pellegrini, \& Tellegen, 1990; Masten \& Powell, 2003). Nesse projeto, o principal instrumento utilizado para mensuração de resiliência foi o Life Events Questionnaire (LEQ), havendo ainda outros instrumentos, usados para avaliar o conhecimento dos pais acerca do comportamento das crianças em casa e na escola, como sejam: Home Rating Scales, Family Rating Scales e Community Mental Health Interview (Masten et al., 1990). Por sua vez, as desordens parentais de personalidade antissocial foram mensuradas através do Antisocial Behavior Inventory (Zucker, Ellis, Fitzgerald, Bingham, \& Sanford, 1996), sendo ainda usada a Parental Bonding Instrument (PBI; Parker, Tupling, \& Brown, 1979), em uma amostra de indivíduos de 16 anos de idade, com o intuito de avaliar a qualidade do cuidado parental. Por último, o projeto envolveu a aplicação do Acceptance and Emotional Verbal Responsivity Subscales of the HOME inventory (Bradley \& Caldwell, 1984) para mensurar a responsividade materna, assim como a Aggression Subscale do Jackson Personality Research Form (Jackson, 1967) e a Overt Anger Subscale do Handling Anger Questionnaire (Spielberger et al., 1985), para avaliar algumas características da personalidade materna.

Um aspecto comum a esses estudos pioneiros é o fato de eles haverem se voltado à investigação de processos de adaptação positiva, uma diferença significativa em relação à tendência científica da época (Masten et al., 1990). Segundo Rutter (2000), durante um longo tempo esses processos haviam recebido pouca atenção, em parte devido a um desinteresse no estudo das diferenças individuais (que tendiam a ser atribuídas a fatores constitucionais indefinidos), ou também porque tais processos pareciam ser de difícil explicação, ou ainda em função do temor de que um direcionamento do foco para desfechos positivos em face de condições adversas pudesse distrair a atenção das autoridades em relação à gravidade dessas condições. Entretanto, as crescentes mudanças no clima científico e a emergência da própria "Psicologia Positiva" vieram a abrir caminho para o estudo dos fenômenos compreendidos pelo conceito que viria a ser denominado resiliência.

Uma das primeiras áreas do conhecimento científico a utilizar o termo "resiliência" para conceituar um determinado fenômeno foi a Física (Yunes, 2003): na área de mecânica dos sólidos, esse conceito refere-se à "propriedade que alguns corpos apresentam de retornar à sua forma original após terem sido submetidos a uma deformação elástica" (Houaiss \& Villar, 2001). Porém, o conceito de resiliência não permaneceu limitado a uma única disciplina, mas passou a compreender uma ideia ampla, referente à "capacidade apresentada por um sistema dinâmico de suportar ou de recuperar-se de distúrbios significativos" (Masten, 2007). Tal conceito genérico foi oportunamente adaptado a contextos específicos de outras áreas do conhecimento, tais como a Ecologia (Holling, 1973), a Economia (Briguglio, Cordina, Farrugia, \& Vella, 2009) 
e a Psicologia, a partir da metáfora originalmente associada ao sentido físico e ao caráter dinâmico denotados por esse termo.

\section{Complexidade do Construto}

Embora o conceito genérico de resiliência venha sendo utilizado em uma variedade de áreas do conhecimento, deve-se observar a existência de importantes diferenças e particularidades relativas a cada contexto específico. Por exemplo, enquanto no campo da Física a resiliência corresponde a uma propriedade de um determinado corpo, no campo da Psicologia e demais ciências do comportamento esse conceito corresponde a um processo de caráter transacional, mediado pela interação entre o indivíduo e o ambiente, cujo resultado varia em função da sinergia formada por diversos atributos e subprocessos (Jaffee, Caspi, Moffitt, Polo-Tomas, \& Taylor, 2007; Masten \& Obradovié, 2006).

Duas categorias distintas de fatores probabilísticos, cujos efeitos potenciais são mutuamente opostos, figuram como componentes de fundamental importância nesse processo: os fatores de risco (tipicamente associados a padrões de adaptação negativa) e os fatores de proteção (tipicamente associados a padrões de adaptação positiva diante de contextos de risco). Ambas as categorias têm sido amplamente investigadas através de estudos comportamentais (Werner, 2000), e compreendem elementos pertinentes a vários domínios do funcionamento humano, tais como atributos pessoais, estrutura e dinâmica familiar, contexto social, aspectos culturais e eventos específicos (Masten \& Obradovié, 2006).

Contudo, embora haja evidências empíricas de que grande parte da diversidade existente entre padrões de adaptação individual possa ser resultante da combinação dos efeitos dos fatores de risco e proteção, o estudo da resiliência não deve estar limitado à investigação de tais elementos. Segundo Rutter (2006), as concepções tradicionais de risco e proteção partem da premissa implícita de que seus respectivos impactos serão bastante similares entre diferentes indivíduos, atribuindo a variabilidade dos resultados observados ao grau de equilíbrio existente entre esses fatores em cada caso particular. $\mathrm{O}$ conceito de resiliência, por outro lado, parte do reconhecimento de que há uma grande variabilidade na forma como os indivíduos respondem a situações e experiências semelhantes. Portanto, um entendimento mais aprofundado dos mecanismos associados a tal variabilidade poderá contribuir significativamente para a definição de estratégias adequadas de prevenção e promoção da saúde.

Dessa forma, Rutter (2006) argumenta que a resiliência não é apenas um novo termo utilizado para referenciar conceitos já existentes. Embora compreenda e requeira a investigação prévia dos fatores de risco e proteção presentes em cada contexto, o conceito de resiliência acrescenta uma nova dimensão ao estudo dos fenômenos influenciados por tais fatores. $\mathrm{O}$ autor observa que um dos mais importantes aspectos relacionados a esse conceito é a promoção de uma mudança significativa de foco em comparação aos conceitos de risco e proteção: em vez de concentrar-se na análise de variáveis que influenciam a adaptação do indivíduo, o estudo da resiliência deve estar concentrado na análise de processos (ou mecanismos) mediadores dessa adaptação, refletindo assim a natureza dinâmica desse construto.

Entre os mecanismos mediadores da resiliência podem estar incluídas estratégias pessoais de coping (Rutter, 2000, 2007), um conceito definido por Lazarus e Folkman (1984) como sendo um conjunto dinâmico de esforços cognitivos e comportamentais usados pelo indivíduo para lidar com demandas internas ou externas, as quais são percebidas como uma sobrecarga em relação aos seus recursos pessoais. Dentro dessa perspectiva, portanto, o coping não equivale simplesmente a um comportamento adaptativo automatizado e, assim como a resiliência, é um fenômeno que corresponde a um processo, sendo mais um estado do que um traço pessoal (Lazarus \& Folkman, 1984). A ampla variabilidade de mecanismos de coping e de seus respectivos resultados diante de diferentes tipos de adversidades indica que é necessário dar-se a devida atenção não apenas a aspectos contextuais ou a atributos e experiências pessoais, mas também a determinadas operações mentais que possam atuar na mediação do processo de adaptação do indivíduo (Rutter, 2007).

Deve-se ter em mente que a resiliência é um fenômeno caracterizado essencialmente pelos seguintes aspectos: (a) a presença de condições adversas significativas, que constituem fatores de risco frequentemente associados à ocorrência de desajustamentos biopsicossociais diversos; e (b) a demonstração de padrões de adaptação positiva diante da exposição do indivíduo aos fatores de risco em questão. Tal caracterização reflete a natureza inferencial desse construto, o qual não pode ser diretamente observado, mas sim indiretamente inferido a partir da análise conjunta dos aspectos anteriormente citados (Luthar, 2006; Masten \& Gerwitz, 2006; Rutter, 2006). Portanto, avaliações baseadas apenas em indicadores de competência e saúde mental, como é o caso de várias escalas que pretendem mensurar resiliência, não estariam efetivamente avaliando a resiliência do indivíduo, em virtude de estarem ignorando um aspecto fundamental desse construto: a existência de risco. Qualquer avaliação efetiva de resiliência deve compreender uma análise adequada não apenas dos padrões adaptativos individuais, mas também das adversidades presentes no contexto. Essa análise deve contemplar uma ampla faixa de possíveis efeitos negativos da exposição ao risco, bem como deve estar baseada em uma medida objetiva do grau de risco apresentado, reduzindo-se assim a possibilidade de formulação de conclusões incorretas em decorrência de falhas metodológicas (Rutter, 2006).

Todavia, a complexidade da resiliência não se resume à existência de diversos aspectos que podem influenciar o processo de adaptação do indivíduo, ou às inúmeras 
formas e proporções em que esses aspectos podem estar combinados. Rutter $(2006,2007)$ apresenta importantes considerações a respeito de diversos outros pontos que ilustram essa complexidade, tais como:

1. A exposição a determinados riscos pode ter efeitos paradoxalmente positivos: evidências empíricas indicam que a exposição a condições adversas pode, em determinadas circunstâncias, aumentar a resistência do indivíduo a experiências semelhantes futuras, de forma análoga ao que ocorre em relação à exposição a agentes infecciosos, que pode resultar na obtenção de certo grau de imunidade em relação a exposições posteriores aos mesmos agentes. Os resultados observados em um estudo longitudinal de coorte que acompanhou a trajetória de indivíduos californianos que enfrentaram a Grande Depressão dos anos 30 (Elder, 1974) constituem um exemplo ilustrativo desse aspecto.

2. Alguns elementos podem produzir efeitos protetores em determinadas circunstâncias de risco, mas podem ser neutros (ou mesmo negativos) na ausência de riscos contextuais: por exemplo, a adoção de uma criança vítima de abuso ou negligência parental pode ter um efeito protetor significativo, enquanto a adoção de uma criança oriunda de um ambiente de baixo risco e de cuidado parental adequado pode não apresentar qualquer vantagem particularmente relevante em relação ao seu desenvolvimento e à sua saúde mental.

3. Os padrões de adaptação individuais podem variar em relação a diferentes tipos de adversidades ou de domínios adaptativos: um mesmo indivíduo pode demonstrar resiliência em relação a determinados tipos de risco, mas não em relação a outros, ou pode demonstrar padrões adaptativos positivos restritos a apenas determinados domínios do funcionamento humano.

4. A resiliência não é uma condição que possa ser considerada estável e constante ao longo do tempo: um indivíduo pode demonstrar diferentes graus de resiliência em diferentes períodos da sua trajetória de vida, oscilações que podem ocorrer, por exemplo, em função de mudanças contextuais significativas.

Certamente, tal grau de complexidade não se restringe apenas à resiliência, sendo também inerente a diversos outros construtos, tais como: autopercepção, afetividade, qualidade de vida, estresse, etc. No amplo cenário da avaliação psicológica, portanto, evidencia-se o papel fundamental do psicólogo na integração de informações diversas, provenientes de múltiplas fontes, possibilitando a obtenção de resultados úteis e fidedignos. Para tanto, obviamente, faz-se necessário um nível apropriado de conhecimento dos instrumentos de avaliação disponíveis, especialmente em relação a quais variáveis estão sendo efetivamente avaliadas através dos testes psicológicos.

\section{Instrumentos para Avaliação da Resiliência}

Face à complexidade do fenômeno e à sua natureza dinâmica e contextual, antecipa-se que a avaliação da resiliência não pode ser fundamentada em um instrumento único ou unidimensional, ou como uma condição estável do sujeito, devendo ser relativizada em relação aos fatores de risco e proteção do contexto. Infelizmente, a generalidade dos instrumentos que se destinam à avaliação do construto resiliência centra-se em variáveis psicológicas envolvidas na resiliência (como apoio social, coping, autoeficácia, ajustamento emocional, etc), mas não propriamente no construto em si. Importa, mesmo assim, descrever os principais instrumentos de avaliação da resiliência disponíveis na investigação psicológica.

A Adolescent Resilience Scale foi desenvolvida por Oshio et al. (2003), sendo composta por 21 itens, avaliando três fatores: busca de novidades, regulação emocional e orientação positiva para o futuro. Foi concebida para jovens japoneses, com a finalidade de mensurar características psicológicas de indivíduos resilientes e o quanto os sujeitos são capazes de manter a saúde mental, sem deterioração, mesmo depois de experimentarem acontecimentos dolorosos na vida. Trata-se de um instrumento de autorrelato, respondido por meio de uma escala Likert de cinco pontos. A escala original apresenta boa consistência interna (alpha da escala total $=0,85$; alphas por fatores: procura de novidades $=0,79$; regulação emocional $=0,77$; e orientação positiva para o futuro $=0,81$ ). A escala apresenta também evidências de validade de critério, uma vez que os escores de grupos considerados bem ajustados foram maiores que os escores do grupo vulnerável.

A Resilience Scale, desenvolvida por Wagnild e Young (1993), avalia o nível de adaptação psicossocial positiva nos acontecimentos significativos da vida. Originalmente, essa escala foi construída a partir do relato de $24 \mathrm{mu}-$ lheres (previamente selecionadas por adaptarem-se com sucesso à adversidade da vida) e destinada à população adulta. O teste é composto por 25 itens, respondidos em uma escala do tipo Likert de sete pontos, onde escores altos indicam elevada resiliência. A escala apresenta evidências de diferentes tipos de validade (conteúdo, construto e critério). Nos estudos de validade de construto dessa medida, cinco componentes foram identificados como fatores para resiliência: serenidade, perseverança, autoconfiança, sentido de vida e autossuficiência.

A Resilience Scale foi adaptada para o português brasileiro pelo grupo de estudos de Pesce et al. (2005), que realizaram também estudos de confiabilidade e validade desse instrumento. Para o estudo de adaptação, foi composta uma amostra de 997 escolares, com idade média de 15,4 anos (desvio-padrão $=1,48$ ), alunos de $7^{\mathrm{a}}$ e $8^{\mathrm{a}}$ séries do Ensino Fundamental e dos $1^{\circ}$ e $2^{\circ}$ anos do Ensino Médio de escolas municipais do Rio de Janeiro. A adaptação conservou os 25 itens originais e apresentou bons 
indicadores de validade de conteúdo (equivalência semântica), de consistência interna (escala total $=0,80$ ) e de confiabilidade intraobservador. O kappa oscilou entre discreto e moderado, com intervalo de confiança de $95 \%$.

A Child Psychosocial Distress Screener (CPDS; Jordans, Komproe, Tol, \& De Jong, 2009) é outro instrumento descrito por seus autores como próprio para avaliação de resiliência. Trata-se de um instrumento de triagem breve, multidimensional e sensível para o uso em emergências. Foi desenvolvido em um programa de atenção psicossocial a crianças de quatro países afetados por conflitos e em situações de violência (Sri Lanka, Indonésia, Burundi e Sudão). A CPDS visa a avaliar o sofrimento psicossocial não-específico e a detecção precoce dos prejuízos emocionais decorrentes. Apresenta como características principais: avaliação de sofrimento nãoespecífico, que auxilia a detecção da psicopatologia; é um instrumento breve e administrável por não-clínicos (composto por sete itens, respondidos em uma escala de três pontos); avalia aspectos contextuais e da comunidade; e permite a triangulação de informações (crianças e professores). Apresenta evidências de validade de construto e de critério (sensibilidade diagnóstica e especificidade moderada: 0,84 e 0,64, respectivamente) para avaliação de sofrimento psicossocial não-específico: aborda questões como sofrimento traumático e atual, percepção de enfrentamento e apoio social e adaptação escolar.

A Connor-Davidson Resilience Scale (CD-RISC; Connor \& Davidson, 2003) aparece utilizada na avaliação de traumas. Trata-se de um instrumento formado por 25 itens autorrespondidos. Cada item é pontuado em uma escala Likert de 5 pontos, que quantifica a resiliência no contexto da exposição ao trauma. Estudos indicam que essa medida apresenta boa confiabilidade e indicadores de validade de conteúdo (Bruwer, Emsley, Kidd, Lochner, $\&$ Seedat, 2008).

Para utilização no campo militar, o Deployment Risk and Resilience Inventory (DRRI; King, King, Vogt, Knight, \& Samper, 2006) é um conjunto de escalas multidimensionais que podem ser utilizadas para avaliar fatores relacionados à saúde e bem-estar dos veteranos militares. Inclui escalas para avaliar dois fatores de précombate (estressores prévios e ambiente familiar na infância), 10 fatores relativos ao combate (experiências de combate; percepção de ameaça; consequências das batalhas; dificuldades da vida e ambiente de trabalho; senso de preparação e exposições nucleares, biológicas e químicas; preocupações sobre a vida e rupturas familiares; apoio social; assédio sexual e assédio em geral) e dois fatores pós-combate (apoio social e estresse). Resultados da administração dessas escalas em veteranos de guerra demonstraram que esse instrumento apresenta alta consistência interna e adequados níveis de confiabilidade no teste-reteste.

Por fim, outro instrumento definido por seus autores como próprio para avaliação de resiliência é a Child Health and Illness Profile-Child Edition (CHIP-CE; Riley et al., 2006). Esse instrumento foi utilizado por Forrest, Riley, Vivier, Gordon e Starfield (2004) para avaliar a saúde de crianças de 6 a 11 anos, a partir da perspectiva das próprias crianças e de seus pais. Esse teste é composto por 76 itens que fornecem um abrangente perfil da qualidade de vida e saúde em cinco domínios: satisfação com a saúde e consigo (11 itens); conforto, sintomas físicos e emocionais, e restrições nas atividades devido a doenças (22 itens); resiliência, comportamentos e participação da família em atividades que auxiliem na melhora da saúde (19 itens); riscos e comportamentos que possam representar risco à saúde (14 itens); e realização e desenvolvimento escolar - 10 itens (Forrest et al., 2004). A versão da CHIP-CE respondida pelos pais é denominada Parent Report Form - Child Health and Illness Profile-Child Edition (Riley et al., 2006), sendo uma medida da saúde e da qualidade de vida da criança através da avaliação de seus pais. Foi desenvolvida e validada na Espanha para pais de crianças de seis a onze anos, havendo estudos que mostram que esse instrumento é válido em diferentes condições étnicas e socioeconômicas (Riley et al., 2006).

\section{Considerações Finais}

A avaliação da resiliência na Psicologia é assunto de controvérsia. Se em algumas pesquisas se avalia a resiliência através de questionários de personalidade e de temperamento pouco específicos, em outras, os recursos utilizados, ainda que chamados de instrumentos para medir a "resiliência", acabam sendo mais instrumentos de medida de ajustamento psicossocial. Face à complexidade do construto, tais escalas de resiliência são criticáveis por pretenderem avaliar um traço psicológico sem a sua forçosa contextualização. Importa reconhecer que os itens das escalas consideram fatores de risco e de proteção que não podem ser generalizados às diversas faixas etárias, quadros socioeconômicos ou grupos culturais.

Portanto, muitos desses instrumentos são úteis para avaliar características estáveis de indivíduos, como traços de personalidade, por exemplo, mas não contemplam satisfatoriamente o construto resiliência. Existe notória variabilidade intraindividual quando um sujeito é submetido a experiências estressoras ou condições adversas que ameaçam o seu bem-estar e saúde mental (Luthar, 2006; Masten, 2007; Rutter, 2006).

Deve-se considerar que, embora alguns dos instrumentos apresentados tenham sido construídos para avaliar estados (situações transitórias, temporárias), ainda assim são instrumentos que avaliam características estáveis de um indivíduo (ansiedade, raiva, serenidade, perseverança, autoconfiança, etc.), as quais podem sofrer alterações em função de eventos de vida ou situações específicas. Resiliência é uma metáfora ou, em termos psicológicos, um estado. Não é um traço de personalidade ou uma propriedade neuropsicológica dos indivíduos. A resiliência descreve o processo resultante de uma situação em que 
um indivíduo enfrenta, com relativo sucesso, condições adversas e situações de risco, com o auxílio de fatores de proteção que podem ser (e frequentemente são) externos e fora de seu controle. A natureza interativa, desenvolvimental e contextual do construto nos diz que ser resiliente em uma situação não garante, nem permite prever, que o indivíduo será novamente resiliente em uma situação semelhante no futuro e, muito menos, resiliente em situações diferentes. Alterações na rede de apoio social, por exemplo, podem ter mais impacto sobre a capacidade de enfrentar com sucesso situações de risco do que variáveis individuais, como ansiedade, depressão, estresse ou autoeficácia, entre outras.

Importa, portanto, um maior investimento da pesquisa na operacionalização da resiliência de forma a reunir os melhores indicadores, por exemplo de registro direto, para a sua avaliação. Com a Psicologia Cognitiva, generalizou-se o recurso a escalas de autorrelato, acreditando-se na capacidade do sujeito de se autoavaliar com precisão nas diferentes dimensões psicológicas, o que pode não ser tão adequado à avaliação de dimensões psicológicas suscetíveis de mudanças no tempo e no contexto. No caso da resiliência, como ressalta Rutter (2006), há ainda a necessidade de uma avaliação apropriada das adversidades presentes ao longo da trajetória do indivíduo, uma vez que só faz sentido falar-se em resiliência diante de contextos que efetivamente apresentem riscos significativos.

Dada a relevância que a resiliência e o seu estudo representam na psicologia dos nossos dias, importaria um maior investimento na construção e validação de instrumentos alternativos e complementares para a sua avaliação. Uma análise efetiva desse construto requer um sistema de avaliação multidimensional, que compreenda os diversos aspectos relacionados à investigação da resiliência como um processo dinâmico, e não apenas como um conjunto de indicadores de competência e ajustamento biopsicossocial. Faz-se necessário, ainda, que a avaliação da resiliência seja devidamente contextualizada em relação a diferentes tipos de risco e domínios adaptativos - não basta apenas que se tenha a informação de que um determinado indivíduo demonstra resiliência, é importante que se saiba como essa resiliência se manifesta, e diante de quais condições adversas.

\section{Referências}

Anthony, E. J. (1974). The syndrome of the psychologically invulnerable child. In E. J. Anthony \& C. Koupernik (Eds.), The child in his family: Children at psychiatric risk (pp. 529545). New York: Wiley.

Anthony, E. J. (1987). Children at high risk for psychosis growing up successfully. In E. J. Anthony \& B. J. Cohler (Eds.), The invunerable child (pp. 147-184). New York: Guilford Press.

Bradley, R. H., \& Caldwell, B. M. (1984). The relation of infants' home environments to achievement test performance in first grade: A follow-up study. Child Development, 55, 803-809.
Briguglio, L., Cordina, G., Farrugia, N., \& Vella, S. (2009). Economic vulnerability and resilience: Concepts and measurements. Oxford Development Studies, 37(3), 229-247.

Bruwer, B., Emsley, R., Kidd, M., Lochner, C., \& Seedat, S. (2008). Psychometric properties of the Multidimensional Scale of Perceived Social Support in youth. Comprehensive Psychiatry, 49, 195-201.

Connor, K. M., \& Davidson, J. R. T. (2003). Development of a new resilience scale: The Connor-Davidson Resilience Scale (CD-RISC). Depression and Anxiety, 18(2), 76-82.

Elder, G. H. (1974). Children of the great depression. Chicago, IL: University of Chicago Press.

Forrest, C. B., Riley, A. W., Vivier, P. M., Gordon, N. P., \& Starfield, B. (2004). Predictors of children's healthcare use: The value of child versus parental perspectives on healthcare needs. Medical Care, 42(3), 232-238.

Hathaway, S. R., \& McKinney, J. C. (1942). The Minnesota Multiphasic Personality Inventory. Minneapolis, MN: University of Minnesota Press.

Holling, C. S. (1973). Resilience and stability of ecological systems. Annual Review of Ecology and Systematics, 4, 1-23. Houaiss, A., \& Villar, M. S. (2001). Dicionário Houaiss da Lingua Portuguesa. Rio de Janeiro, RJ: Objetiva.

Jackson, D. N. (1967). Manual for the personality research form. London: University of Western Ontario.

Jaffee, S. R., Caspi, A., Moffitt, T. E., Polo-Tomas, M., \& Taylor, A. (2007). Individual, family, and neighborhood factors distinguish resilient from non-resilient maltreated children: A cumulative stressors model. Child Abuse \& Neglect, 31(3), 231-253.

Janes, C. L., Gilpin, D., Lander, H., \& Finn, S. (1975). The General Information Questionnaire. Unpublished manuscript.

Jones, F. H. (1977). The Rochester Adaptive Behavior Inventory: A parallel series of instruments for assessing social competence during early and middle childhood and adolescence. In J. S. Strauss, H. M. Babigian, \& M. Roff (Eds.), The origins and course of Psychopathology (pp. 249281). New York: Plenum Press.

Jordans, M. J. D., Komproe, I. H., Tol, W. A., \& De Jong, J. T. V. M. (2009). Screening for psychosocial distress amongst war-affected children: Cross-cultural construct validity of the CPDS. Journal of Child Psychology and Psychiatry, 50(4), 514-523.

King, L. A., King, D. W., Vogt, D. S., Knight, J., \& Samper, R. E. (2006). Deployment risk and resilience inventory: A collection of measures for studying deployment-related experiences of military personnel and veterans. Military Psychology, 18(2), 89-120.

Lazarus, R. S., \& Folkman, S. (1984). Stress, appraisal, and coping. New York: Springer.

Luthar, S. S. (2006). Resilience in development: A synthesis of research across five decades. In D. Cicchetti \& D. J. Cohen (Eds.), Developmental Psychopathology: Risk, disorder and adaptation (pp. 739-795). New York: Wiley.

Masten, A. S. (2007). Resilience in developing systems: Progress and promise as the fourth wave rises. Development and Psychopathology, 19, 921-930.

Masten, A. S., \& Gewirtz, A. H. (2006). Vulnerability and resilience in early child development. In K. McCartney \& D. A. Phillips (Eds.), The Blackwell handbook of early childhood development (pp. 22-43). Oxford, UK: Blackwell. 
Masten, A. S., Morison, P., Pellegrini, D., \& Tellegen, A. (1990). Competence under stress: Risk and protective factors. In J. Rolf, A. S. Masten, D. Cicchetti, K. H. Nuechterlein, \& S. Weintraub (Eds.), Risk and protective factors in the development of Psychopathology (pp. 236-256). New York: Cambridge University Press.

Masten, A. S., \& Obradovié, J. (2006). Competence and resilience in development. Annals of the New York Academy of Sciences, 1094, 13-27.

Masten, A. S., \& Powell, J. L. (2003). A resilience framework for research, policy, and practice. In S. S. Luthar (Ed.), Resilience and vulnerability: Adaptation in the context of childhood adversities (pp. 1-25). New York: Cambridge University Press.

Oshio, A., Kaneko, H., Nagamine, S., \& Nakaya, M. (2003). Construct validity of the Adolescent Resilience Scale. Psychological Reports, 93, 1217-1222.

Parker, G., Tupling, H., \& Brown, L. B. (1979). A Parental Bonding Instrument. British Journal of Medical Psychology, 52, 1-10.

Pesce, R. P., Assis, S. G., Avanci, J. Q., Santos, N. C., Malaquias, J. V., \& Carvalhaes, R. (2005). Adaptação transcultural, confiabilidade e validade da escala de resiliência. Caderno de Saúde Pública, 21(2), 436-448.

Riley, A. W., Coghill, D., Forrest, C. B., Lorenzo, M. J., Ralston, S. J., \& Spiel, G. (2006). Validity of the health-related quality of life assessment in the ADORE study: Parent Report Form of the CHIP - Child Edition. European Child \& Adolescent Psychiatry, 15(1), 63-71.

Rutter, M. (2000). Resilience reconsidered: Conceptual considerations, empirical findings, and policy implications. In J. P. Shonkoff \& S. J. Meisels (Eds.), Handbook of early childhood intervention ( $2^{\text {nd }}$ ed., pp. 651-682). New York: Cambridge University Press.

Rutter, M. (2006). Implications of resilience concepts for scientific understanding. Annals of the New York Academy of Sciences, 1094, 1-12.

Rutter, M. (2007). Resilience, competence and coping. Child Abuse and Neglect, 31, 205-209.

Spielberger, C. D., Johnson, E. H., Russell, S. F., Crane, R. J., Jacobs, G. A., \& Worden, T. J. (1985). The experience and expression of anger: Construction and validation of an anger expression scale. In M. A. Chesney \& R. H. Rosenman (Eds.), Anger and hostility in cardiovascular and behavioral disorders (pp. 5-30). New York: Hemisphere.

Wagnild, G. M., \& Young, H. M. (1993). Development and psychometric evaluation of the Resilience Scale. Journal of Nursing Measurement, 1, 165-168.

Werner, E. E. (1993). Risk, resilience, and recovery: Perspectives from the Kauai longitudinal study. Development and Psychopathology, 5, 503-515.

Werner, E. E. (2000). Protective factors and individual resilience. In J. P. Shonkoff \& S. J. Meisels (Eds.), Handbook of early childhood intervention ( $2^{\text {nd }}$ ed., pp. 115-132). New York: Cambridge University Press.

Werner, E. E., Bierman, J. M., \& French, F. E. (1971). The children of Kauai: A longitudinal study from the prenatal period to age ten. Honolulu, HI: University of Hawaii Press.

Werner, E. E., \& Smith, R. S. (1977). Kauai's children come of age. Honolulu, HI: University of Hawaii Press.

Werner, E. E., \& Smith, R. S. (1982). Vulnerable but invincible: A longitudinal study of resilient children and youth. New York: McGraw-Hill.
Werner, E. E., \& Smith, R. S. (1992). Overcoming the odds: High risk children from birth to adulthood. Ithaca, NY: Cornell University Press.

Yunes, M. A. M. (2003). Psicologia positiva e resiliência: O foco no indivíduo e na família. Psicologia em Estudo, 8, 7584.

Zucker, R. A., Ellis, D. A., Fitzgerald, H. E., Bingham, C. R., \& Sanford, K. P. (1996). Other evidence for at least two alcoholisms, II: Life course variation in antisociality and heterogeneity of alcoholic outcome. Development and Psychopathology, 8, 831-848.
Recebido: $27 / 05 / 2010$

$1^{a}$ revisão: 16/03/2011 Aceite final: 29/03/2011 